\title{
Flowering events in relation to smut susceptibility in pearl millet
}

\author{
R. P. THAKUR \\ Cereals Program, International Crops Research Institute for the Semi-Arid Tropics (ICRISAT). \\ Patancheru. Andhra Pradesh 502 324, India.
}

In field experiments at ICRISAT, selected lines of pearl millet (Pennisefum glaucum) resistant and susceptible to smut (Tolyposporium penicillariae) were evaluated for the timing of flowering events. In smut-resistant lines, the time from the boot-leaf stage (inoculation) to stigma emergence varied in the range 46-120 h, from boot to anther emergence 105-190 h, and from stigma emergence to anther emergence (protogyny period) $59-131 \mathrm{~h}$; in smut-susceptible lines, the corresponding periods were 62$140 \mathrm{~h}, 146-200 \mathrm{~h}$ and $44-120 \mathrm{~h}$, respectively. There were no significant correlations between timing of events and smut severity. Three cytoplasmically male-sterile lines showed longer protogyny periods and higher smut severity than their corresponding maintainer lines. Four lines having short protogyny periods (22-52 h) and resistance to ergot (Claviceps fusiformis) also showed high resistance to smut. Resistance to smut in most ergot-susceptible lines was independent of the timing of flowering events. but in ergot-resistant lines it could be closely related to flowering events.

\section{INTRODUCTION}

Flowering in pearl millet (Pennisetum glaucum) is protogynous, i.e. the stigmas emerge and mature before the stamens. This characteristic of the species makes it highly cross-pollinated, but predisposes it to infecton by ovary-infecting fungal pathogens. Tolyposporium penicillariae. the causal agent of smut, infects the ovary through the young emerging stigma (Bhatt. 1946). Pollination of panicles inoculated with $T$. penicillariae has been shown to reduce smut infection (Thakur et al., 1983a; Wells et al., 1987), and it is generally believed that cross-pollination prevents infection under natural conditions. In a field-based smut screening technique (Thakur et al., 1983b), cross-pollination is prevented by covering the panicles with parchment bags immediately after inoculation at the boot-leaf stage. and removing the bags 20 days after inoculation when development of smut sori and seeds are complete. This technique has been used successfully to identify a number of lines highly resistant to smut at ICRISAT and other locations (Thakur et al., 1986). The bagging of inoculated panicles,

Submitted as ICRISAT Journal Article No. 856. however, does not eliminate self-pollination which may be involved in reduced smut infection.

In ergot disease of pearl millet, caused by Clariceps fusiformus, early pollination of inoculated panicles has been shown to reduce infection (Thakur \& Williams, 1980), and the screening technique designed to prevent cross-pollination (Thakur et al., 1982) has been used successfully in identifying sources of resistance. This resistance is apparently based on short protogyny, which results in rapid self-pollination in the inoculated. bagged panicles. In pearl millet panicles, stigma withering initiates within $3 \mathrm{~h}$, and complete withering occurs within $6 \mathrm{~h}$ after pollination (Thakur \& Williams, 1980; Willingale et al., 1986). The withering of stigmas is caused by the development of a lacalized constriction in the basal tissue of the style within $6 \mathrm{~h}$ of pollination. thus isolating the ovary from the stigma and preventing subsequent infection by $C$. fusiformis (Willingale et al., 1986). The mechanism of resistance to $T$. penicillariae is, however, not clear. In resistance screening at ICRISAT, all ergot-resistant lines (having short protogyny) showed high levels of resistance to smut (Thakur et al., 1985; Thakur \& King. 1988a), but none of the smut-resistant lines showed resistance to ergot (R. P. Thakur, unpublished). This study was 
planned to determine the relationship between the time course of flowering events and susceptibility to smut in selected lines of pearl millet.

\section{MATERIALS AND METHODS}

Field experiments were conducted during the 1985 dry and rainy seasons, and the 1987 and 1988 rainy seasons at ICRISAT. During the 1985 dry and rainy seasons eight pearl millet lines, five resistant to smut ( $<5 \%$ mean severity) and three susceptible ( $>5 \%$ mean severity) were grown, each in a two-row plot, $4 \mathrm{~m}$ long with 20 plants per row. Observations on flowering events were taken during the dry season, and on smut and ergot severities during the rainy season. In the 1987 rainy season, six pearl millet lines, four resistant to ergot ( $<5 \%$ mean severity) and two susceptible ( $>5 \%$ mean severity) were grown, each in a two-row plot. $4 \mathrm{~m}$ long with three replications in a randomized block design, for observations on protogyny and reactions to ergot and smut. During the 1988 rainy season 12 pearl millet lines, eight smut susceptible-including three cytoplasmically male-sterile $(A)$ lines with their corresponding maintainer (B) lines-and four smut-resistant inbreds were grown, each in a two-row plot, $4 \mathrm{~m}$ long with two replications in a randomized block design, for observations on flowering events, smut reaction and seed set.

\section{Observations of flowering events}

In each plot, panicles of 10 plants in the $1985 \mathrm{dry}$ season and the 1988 rainy season and 20 plants in the 1987 rainy season were covered with parchment pollination bags at the boot-leaf stage (boot) with date and time of bagging noted on the bag, and also on the data record sheet. On each panicle, by briefly opening the bag daily at 09.00 and $16.00 \mathrm{~h}$, the time from the boot to initiation of stigma emergence, and to anther emergence was assessed.

\section{Smut inoculation}

In each plot the main shoot panicles of 20 plants in the 1985 and 1987 rainy season and 10 in the 1988 rainy season were inoculated by injecting a suspension of $T$. penicillariue sporidia $\left(10^{6}\right.$ sporidia per $\mathrm{ml}$ ) into the boot leaf sheath, and covering the panicless with parchment bags (Thakur 't al., 1983b). High humidity, essential for infection and disease development, was created by operating an overhead sprinkler for $30 \mathrm{~min}$ between 12.00
13.00 and $17.00-18.00 \mathrm{~h}$, on dry days. Twenty days after inoculation, bags were removed and panicles were scored for smut infection by reference to a smut severity rating scale (Thakur \& King, 1988b). In the 1988 rainy season experiment, observations on flowering events and seed set were recorded in each plot on the same panicles used for smut inoculation, while in other experiments different plants were used.

\section{Ergot inoculation}

In each plot, plants were inoculated at the full stigma emergence stage with a suspension containing $10^{6}$ conidia per $\mathrm{ml}$ of $C$. fusiformis $(10$ plants in the 1985 rainy season and 20 plants in the 1987 rainy season). Panicles were protected from cross-pollination by covering them with parchment bags (Thakur et al., 1982). High humidity was maintained as for smut inoculation. Bags were removed 15 days after inoculation and ergot was assessed with the aid of a severity rating scale (Thakur \& Williams, 1980).

\section{RESULTS}

Time from boot to stigma emergence and smut severity

The time from inoculation at the boot stage to the estimated time for initiation of infection (stigma emergence) varied among the lines. In smutresistant lines it varied from 46 to $91 \mathrm{~h}$ during the dry season (Table I) and from 65 to $120 \mathrm{~h}$ in the rainy season (Table 2 ), and in smut-susceptible lines this period varied from 62 to $84 \mathrm{~h}$ in the dry season and 63 to $140 \mathrm{~h}$ in the rainy season. The smut-resistant lines ICMPS 100-5-1 and ICMPS 900-9-3, common to the two tests, recorded boot to stigma emergence periods of 64 and $89 \mathrm{~h}$ in the dry season, and 65 and $79 \mathrm{~h}$, respectively, in the rainy season. Although there were significant differences between lines, the resistant and susceptible lines could not be discerned on this basis. and there was no significant correlation between the mean value for boot to stigma emergence time and the mean smut severity.

\section{Time from boot to anther emergence and smut severity}

In smut-resistant lines the time from boot to anther emergence varied from 105 to $190 \mathrm{~h}$, and in smut-susceptible lines from 174 to $186 \mathrm{~h}$ during the dry scason (Table 1) and from 132 to $189 \mathrm{~h}$ in 
Table 1. Average period of flowering events, smut severity and ergot severity in selected pearl millet lines. ICRISAT, 1985

\begin{tabular}{|c|c|c|c|c|c|}
\hline \multirow[b]{2}{*}{ Line } & \multicolumn{2}{|c|}{ Time $(h)^{\mathrm{H}}$} & \multirow{2}{*}{$\begin{array}{c}\text { Protogyny } \\
\text { period } \\
(h)^{j}\end{array}$} & \multirow{2}{*}{$\begin{array}{l}\text { Smut } \\
\text { severity } \\
(\%)^{\mathrm{b}}\end{array}$} & \multirow{2}{*}{$\begin{array}{r}\text { Ergot } \\
\text { severit } \\
(\%)\end{array}$} \\
\hline & Boot-SE & Boot-AE & & & \\
\hline \multicolumn{6}{|l|}{ Smut resistant } \\
\hline ICMPS 100-5-1 & 64 & 151 & 87 & 0 & 43 \\
\hline ICMPS 100-5-3-3 & 59 & 190 & 131 & 0 & 75 \\
\hline ICMPS $900-5-4-14$ & 46 & 105 & 59 & 0 & 86 \\
\hline ICMPS $900-9-3$ & 89 & 168 & 79 & 0 & 60 \\
\hline ICMPS 1600-2-9-1 & 91 & 190 & 99 & 0 & 79 \\
\hline \multicolumn{6}{|l|}{ Smut susceptible } \\
\hline BJ 104 & 62 & 174 & 111 & 90 & 94 \\
\hline $5141 \mathrm{~A}$ & 84 & 186 & 102 & 91 & 92 \\
\hline ICH 206 & 78 & 179 & 100 & 85 & 90 \\
\hline SE & $5 \cdot 7$ & $10 \cdot 1$ & 7.6 & $16 \cdot 2$ & $6 \cdot 4$ \\
\hline
\end{tabular}

Based on mean of 10 panicles during the 1985 season.

based on mean of 20 inoculated panicles in the 1985 rainy season smut nursery.

c Based on mean of 10 inoculated panicles in the 1985 rainy season ergot nursery.

SE, stigma emergence; AE, anther emergence; protogyny period = time between $\mathrm{SE}$ and $\mathrm{AE}$.

Table 2. Average period of flowering events, smut severity and seed set in selected pearl millet lines. ICRISAT, rainy season 1988

\begin{tabular}{|c|c|c|c|c|c|}
\hline \multirow[b]{2}{*}{ Line } & \multicolumn{2}{|c|}{ Time $(h)^{\mathrm{a}}$} & \multirow{2}{*}{$\begin{array}{c}\text { Protogyny } \\
\text { period } \\
(h)^{+}\end{array}$} & \multirow{2}{*}{$\begin{array}{c}\text { Smut } \\
\text { severity } \\
(\% / 1)^{\mathrm{d}}\end{array}$} & \multirow{2}{*}{$\begin{array}{l}\text { Seed } \\
\text { set } \\
(\%)^{a}\end{array}$} \\
\hline & Boot-SE & Boot-AE & & & \\
\hline \multicolumn{6}{|l|}{ Smut resistant } \\
\hline SRB 3 & 76 & 132 & 60 & 4 & 86 \\
\hline ICMPS $900-9-3$ & 79 & 170 & 91 & 0 & 73 \\
\hline ICMPS 100-5-1 & 65 & 143 & 78 & 0 & 79 \\
\hline ICMSR II & 120 & 189 & 71 & 0 & 84 \\
\hline \multicolumn{6}{|l|}{ Smut susceptible } \\
\hline $841 \mathrm{~A}$ & 86 & 159 & 74 & 90 & $<1$ \\
\hline $841 B$ & 79 & 146 & 67 & 47 & 39 \\
\hline $81 \mathrm{~A}$ & 82 & 200 & 118 & 88 & $<1$ \\
\hline $81 \mathrm{~B}$ & 90 & 190 & 101 & 70 & 7 \\
\hline $842 A$ & 63 & 183 & 120 & 63 & 0 \\
\hline $842 B$ & 66 & 155 & 90 & 13 & 6 \\
\hline SRB 1 & 80 & 170 & 90 & 10 & 73 \\
\hline SR B 2 & 140 & 183 & 44 & 12 & 63 \\
\hline SE & $5 \cdot 2$ & $7 \cdot 4$ & 3.9 & $2 \cdot 8$ & 2.9 \\
\hline
\end{tabular}

- Mean of 10 panicles in each of two replications; the same panicles were used for recording flowering events and smul inoculations.

SE, stigma emergence; $A E$, anther emergence; protogyny period = time between $S E$ and $A E$. 
smut-resistant lines and 146 to $200 \mathrm{~h}$ in smutsusceptible lines in the rainy season (Table 2 ). The smut-resistant lines ICMPS 100-5-1 and ICMPS 900-9-3 recorded mean times of 151 and $168 \mathrm{~h}$ in the dry season, and 143 and $170 \mathrm{~h}$ in the rainy season, respectively. Although there were significant differences among lines for this trait, there was no significant correlation of the mean period between boot and anther emergence with the mean smut severity.

\section{Protogyny and smut severity}

The mean protogyny period varied from 59 to 131 $\mathrm{h}$ in the dry season and 71 to $91 \mathrm{~h}$ in the rainy season in smut-resistant lines, and from 100 to $111 \mathrm{~h}$ in the dry season and 44 to $120 \mathrm{~h}$ in the rainy season in smut-susceptible lines (Tables 1 and 2). There were significant differences among lines for this trait, but there was no significant correlation between the mean protogyny period and the mean smut severity levels.

Two of the $\mathrm{A}$ lines, $81 \mathrm{~A}$ and $842 \mathrm{~A}$, exhibited significantly longer protogyny periods $(74-120 \mathrm{~h})$ and higher smut severities $(63-90 \%)$ than their corresponding $B$ lines, which had the protogyny periods of $67-101 \mathrm{~h}$ and smut severities of 13$70 \%$ (Table 2). Among the three A B pairs, 842A/ $B$ was least susceptible, although it did not show the shortest mean protogyny period.

\section{Ergot severity}

Ergot severity in smut-resistant or smut-susceptible lines with protogyny periods of $59-131 \mathrm{~h}$ varied from 43 to $92 \%$ (Table 1). Four ergotresistant lines ( $<5 \%$ mean severity) with mean protogyny periods of 22-52 $\mathrm{h}$ all showed complete resistance to smut, while the two ergotsusceptible lines ( $>5 \%$ mean severity) BJ 104 and BK 560, with 91 and $94 \mathrm{~h}$ mean protogyny periods, respectively, showed high susceptibility (80-85\% severity) to smut (Table 3 ).

\section{Seed set}

Seed set was generally higher in smut-resistant lines $(79-86 \%)$ than in smut-susceptible lines (7$73 \%$ ) (Table 2). Two moderately smut-susceptible lines, SRB $1\left(10^{\circ}\right.$ o severity) and SR B 2 (12\% severity), had 73 and $63 \%$ seed set, respectively. There was, however, no significant correlation between the mean protogyny period and the mean seed set.

\section{DISCUSSION}

A generalized time course of flowering events with the smut inoculation and symptom development timings superimposed is shown in Fig. 1. From the time of inoculation at the boot-leaf stage, it takes 3-5 days for $T$. penicillariae sporidia to infect the young stigmas emerging from the glumes of the florets (Bhatt, 1946). During this period, any change in the environment that is unfavourable for sporidial survival might lead to reduced infection. Infection is probably completed in the period from the boot stage to anther emergence and most stigmas would wither in bagged, inoculated panicles; stigmas that remain fresh could receive pollen to facilitate fertilization and thereby prevent smut infection (Thakur et al., 1983a; Wells et al., 1987). In pearl millet, the time course of flowering events could vary particularly with temperature, relative humidity, and the plant phenotype. In the normal growing season a cultivar takes 15-21 days from the boot-leaf stage to the visible grain filling stage, while smut sori appear within 10-14 days of inoculation at boot. Of the three measured intervals between flowering events, boot to stigma emergence, boot to anther emergence, and stigma emergence to anther emergence (protogyny), protogyny seems to be the most important in the cultivars for which smut resistance is related to pollination.

The results indicated that resistance to smut in ergot-resistant cultivars is probably associated with self-pollination and fertilization, but in other cultivars it is not associated with self-pollination. Under natural conditions, escape from smut infection can be attributed to cross-pollination (Thakur et al., 1983a), as with ergot (Thakur \& Williams, 1980; Thakur et al., 1983d).

Thakur et al. (1983a) and Wells et al. (1987) demonstrated that smut-inoculated panicles when pollinated at full-stigma emergence, i.e. 3-4 days after inoculation, showed significantly less smut infection. This indicates that smut infection can be enhanced in the absence of pollen, and suggests that lines with a longer protogyny period would be more susceptible. This hypothesis is supported by the fact that ergot-resistant lines. which generally have shorter protogyny than ergot-susceptible lines, have shown high levels of smut resistance. Conversely, all the smut-resistant lines that do not have short protogyny are highly susceptible to ergot (R. P. Thakur, unpublished). In this study, smut-resistant lines with $59-$ $132 \mathrm{~h}$ protogyny recorded $43.86 \%$ ergol severity 


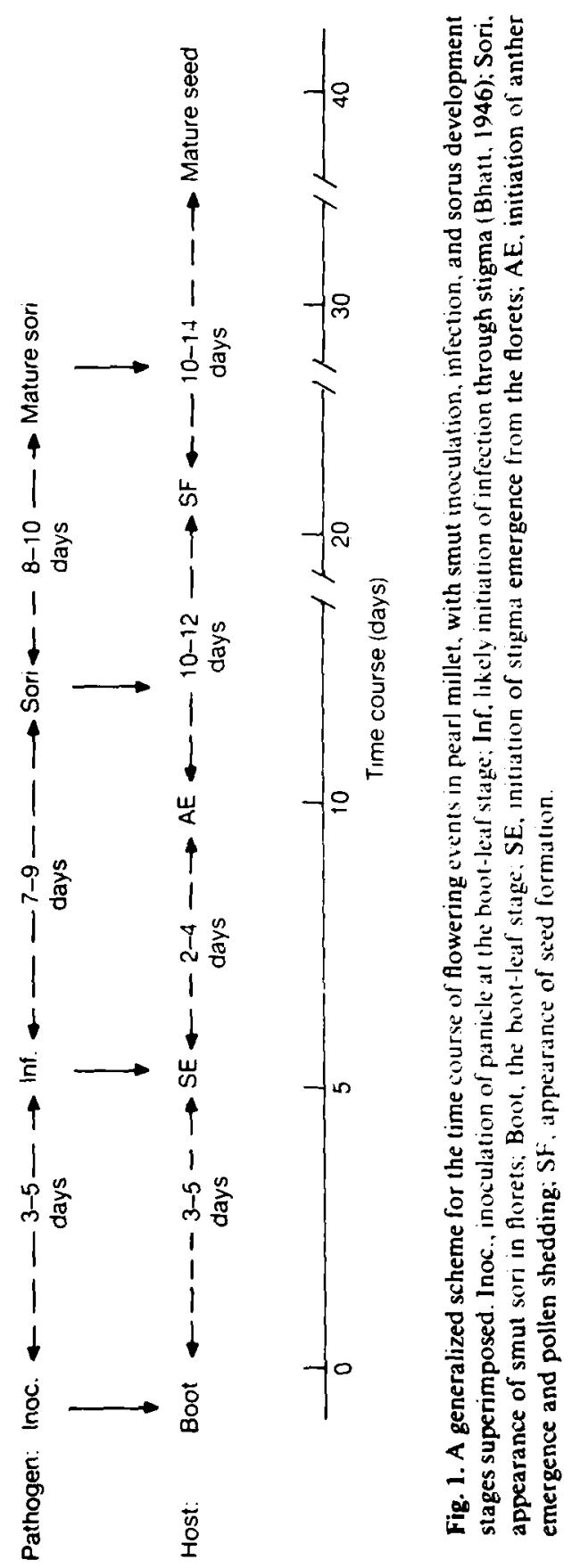


Table 3. Protogyny period, smut severity and ergot severity of pearl millet lines resistant and susceptible to ergot, ICRISAT, rainy season 1987

\begin{tabular}{lccc}
\hline Line & $\begin{array}{c}\text { Protogyny } \\
\text { period } \\
(\mathrm{h})^{2}\end{array}$ & $\begin{array}{c}\text { Ergot } \\
\text { severity } \\
(\%)^{2}\end{array}$ & $\begin{array}{c}\text { Smut } \\
\text { severity } \\
(\%)^{\mathbf{a}}\end{array}$ \\
\hline Ergot resistant & & & \\
ICMPES 1 & 22 & $<1$ & 0 \\
ICMPES 2 & 52 & $<1$ & 0 \\
ICMPES 29 & 43 & $<1$ & 0 \\
ICMPES 34 & 37 & 4 & 0 \\
Ergot susceptible & & & \\
BJ 104 & 94 & 92 & 80 \\
BK 560 & 91 & 94 & 85 \\
SE & 12.0 & 10.8 & 17.4 \\
\hline
\end{tabular}

Based on mean of 20 panicles in each of three replications.

(Table 1), while ergot-resistant lines with 22-52 h protogyny showed complete resistance to smut (Table 3). From this, it seems that lines with a protogyny period of more than $52 \mathrm{~h}$ are likely to be susceptible to ergot, but not to smut because protogyny periods in smut-resistant lines were 59-131 h. In 1985, timings of flowering events were recorded in the dry season, and smut and ergot scorings were taken in the rainy season, but the results of 1985 are in agreement with the 1988 rainy season data for the two common lines, ICMPS 100-5-1 and ICMPS 900-9-3, indicating insignificant effect of seasons on the timings of flowering events.

The longer protogyny and higher smut severity of $A$ lines could be associated with the cytoplasmic male-sterility system; in B lines, production of pollen and partial self-pollination under bagged panicles might account for reduced smut infection. High smut susceptibility of most of the $F_{1}$ hybrids could be attributed to their malesterile cytoplasm. In a study with five $A$ lines and their corresponding $B$ lines, and hybrids made on them. Khairwal 't al. (1986) reported no significant difference for smut susceptibility between hydrids made with $A$ and $B$ lines, and the lower smut susceptibility of $B$ lines than of $A$ lines was attributed to rapid pollination in $B$ lines. They concluded that male-sterile cytoplasm was not related to smut susceptibility of the hybrids. However, they did not provide data on protogyny and seed set of the parental lines and the hybrids. In other lines the period of prologyny was not correlated with smut severity levels. It could be argued that lines with short protogyny would have good seed set under selfing and would have resistance to smut. Smut resistance, however, is not associated with good seed set; a number of highly smut-resistant lines with very few or no seed set under selfing were observed. It seems that the amount of seed set depends on several factors that are not related to smut resistance.

Smut epidemics in pearl millet are unlikely because the relatively long latent period (about 20 days) drastically reduces the chance of secondary infection in a crop, and the occurrence under natural conditions of cross-pollination reduces smut infection. This hypothesis is well supported by the fact that so far there has not been even a single report of an epidemic, despite the survival of the pathogen in most areas of the world where pearl millet is grown (Rachie \& Majmudar, 1980; Thakur \& King, 1988b). Smut in severe form does occur, however, when cross-pollination is reduced during the rainy period at flowering. Then the $F_{1}$ hybrids with their more synchronous tillering and flowering generally develop more smut than the heterogeneous open-pollinated varieties.

Because of increasing commercial cultivation of $F_{1}$ hybrids in India, resistance to smut has become an important component of the hybrid breeding programme at ICRISAT. A number of pearl millet lines that have high levels of resistance to smut (but not to ergot) and desirable agronomic traits have been identified (Thakur \& Chahal, 1987: Thakur \& King, 1988b), and several of these are being utilized in the programme. Recent studies on inheritance of smut resistance have indicated that resistance is controlled by a few dominant genes with additive effects (Chavan et al., 1988). Utilization of smut resistance based on short protogyny might be difficult to transfer as the inheritance of resistance could be complex. like that reported for ergot (Thakur et al., 1983c).

\section{ACKNOWLEDGEMENTS}

I wish to thank V. P. Rao for his help in conducting the field experiments and data analysis, and S. B. King for his comments on the manuscript.

\section{REFERENCES}

Bhatt R.S. (1946) Studies in ('stiluginales. 1. The mode of infection of the bajos plant (Pennisefum nphoides 
Stapf \& Hubbard) by the smut. Tolyposporium penicillariae Bref. Journal of Indian Botanical Society' 25, 163-186.

Chavan S.B., Thakur R.P. \& Rao K.P. (1988) Inheritance of smut resistance in pearl millet. Plant Disease Research 3, 192-197.

Khairwal, I.S., Singh, S. \& Prakash, Om. (1986) Smut reactions of pearl millet lines and hybrids with and without cytoplasmic male sterility. Proceedings of the Indian National Science Academy B52(6), 751-754.

Rachie K.O. \& Majmudar J.V. (1980) Pearl Millet. Pennsylvania University Press, University Park.

Thakur R.P. \& Chahal S.S. (1987) Problems and strategies in the control of ergot and smut in pearl millet. In: Proceedings of the International Pearl Millet Workshop, ICRISAT. 7-II April 1986, pp. 173-182. ICRISAT. Patancheru.

Thakur R.P. \& King S.B. (1988a) Ergot disease of pearl millet. Information Bulletin No. 24, 24 pp. ICRISAT, Patancheru.

Thakur R.P. \& King S.B. (1988b) Smut disease of pearl Millet. Information Bulletin No. 25, 17 pp. ICRISAT, Patancheru.

Thakur R.P., Rao V.P., Williams R.J., Chahal S.S., Mathur S.B., Pawar N.B., Nafade S.D., Shetty H.S., Singh G. \& Bangar S.G. (1985) Identification of stable resistance to ergot in pearl millet. Plant Disease 69, 982-985.

Thakur R.P., Subba Rao K.V. \& Williams R.J. (1983a) Effects of pollination on smut development in pearl millet. Plant Pathology 32, 141-144.
Thakur R.P., Subba Rao K.V. \& Williams R.J. (1983b) Evaluation of a new field screening technique for smut resistance in pearl millet. Phytopothology 73. $1255 \cdot 1258$.

Thakur R.P.. Subba Rao K.V., Williams R.J., Gupta S.C. Thakur D.P.. Nafade S.D., Sundaram N.V., Frowd J.A. \& Guthrie J.E. (1986) Identification of stable resistance to smut in pearl millet. Plani Disease 70, $38-41$

Thakur R.P., Talukdar B.S. \& Rao V.P. (1983c) Genetics of ergot resistance in pearl millet. Abstracts, I5th International Congress of Genetics, New Delhi. India, abstract No. 1336.

Thakur R.P. \& Williams R.J. (1980) Pollination effects on pearl millet ergot. Phyropathology 70, 80-84.

Thakur R.P., Williams R.J. \& Rao V.P. (1982) Development of resistance to ergot in pearl millet. Phyropathology 72, 406408.

Thakur R.P.. Williams R.J. \& Rao V.P. (1983d) Control of ergot in pearl millet through pollen management. Annals of .Applied Biology 103, 31-36.

Wells. H.D., Hanna, W.W. \& Burton. B.W. (1987) Effects of inoculation and pollination on smut development in near-isogenic lines of pearl millet. Phylopathology 77, 293-296.

Willingale J., Mantle P.G. \& Thakur R.P. (1986) Postpollination stigmatic constriction, the basis for ergot resistance in selected lines of pearl millet. Phrtopathology 76, 536-539. 
This document is a scanned copy of a printed document. No warranty is given about the accuracy of the copy. Users should refer to the original published version of the material. 